\title{
Microfluidic Behavior of Alumina Nanotube-Based Pathways within Hydrophobic CNT Barriers
}

Cemile Aksu', Philip D. Bradford ${ }^{1}$, and Jesse S. Jur ${ }^{1 *}$

${ }^{1}$ Department of Textile Engineering, Chemistry and Science, North Carolina State University, Raleigh, North Carolina 27695-8301, United States

\section{Supporting Information}

The resistance change during microfluidic experiments was measured by real-time data acquisition device (NI USB-6009 DAQ) with a frequency of 50 samples per second. The DAQ device acted as an interface between the computer and the microfluidic device and the data was sent to MATLAB ${ }^{\circledR}$ via DAQ device for real-time plotting and analysis. For each case, the experiments were repeated three times.
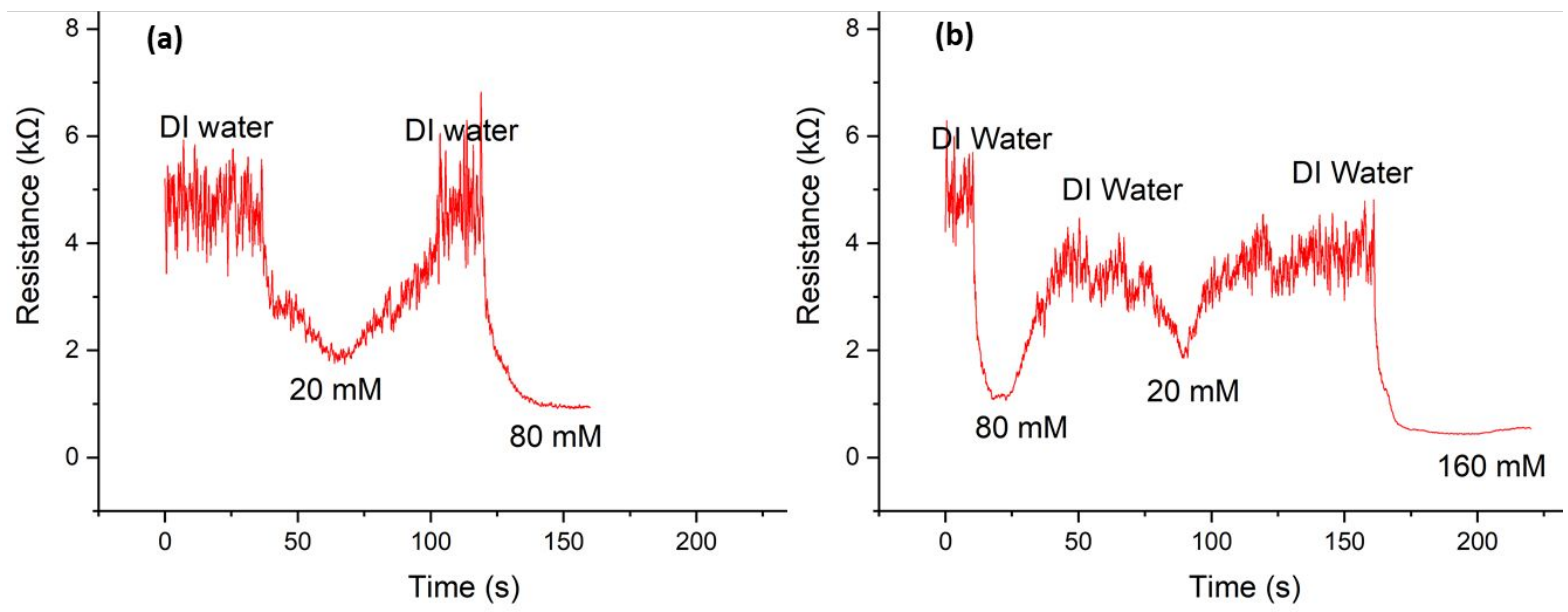

Figure S1. Plots of resistance response of the microfluidic devices with successive feeding of DI water and $\mathrm{NaCl}$ solutions with varying DI water rinse times. 
The resistance response of the microfluidic device to changes in $\mathrm{NaCl}$ solution concentrations is shown in Figure S1. The meter used for this set of experiments had a maximum sensitivity of $5 \mathrm{~K}$ ohms, therefore the data points approaching $5 \mathrm{~K}$ ohms should be considered insulating. Due to the fact that DI water lacks ions, it is not electrically conductive. As expected, the resistance response of the alumina channel does not change after DI water flow within the channel and fluctuates at the saturation value of the meter. Figure S1a shows that, when DI water stayed in the channel for longer times, the resistance increased back to the original resistance indicating that there is no ion entrapment in the alumina nanotubes. These results were confirmed with EDS analysis on the SEM as in Figure S2. However due to the lower DI rinse time represented in Figure S1b, there was not enough time for the DI water to return to the original resistance.

(a)

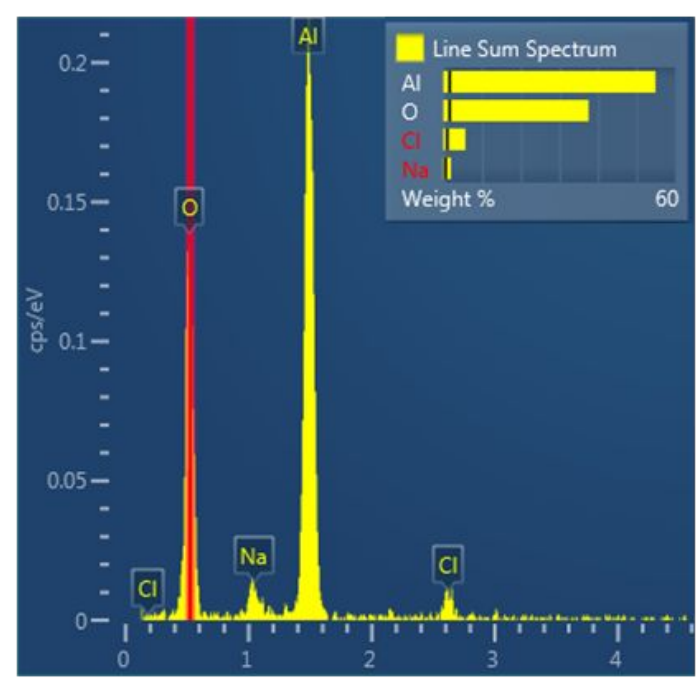

(b)

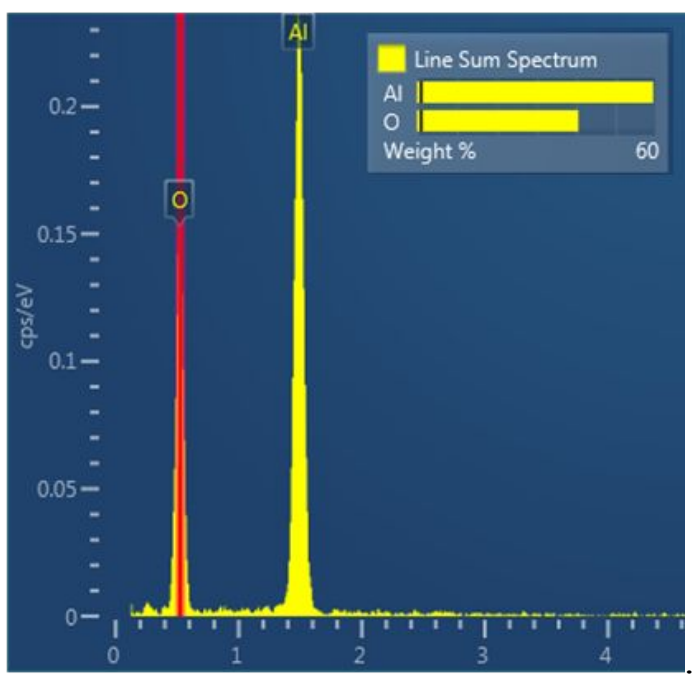

Figure S2. EDS analyses of alumina nanotube channel with (a) $\mathrm{NaCl}$ solution and (b) following DI water rinse. 
Figure S2a shows the EDS analysis of an alumina nanotube channel treated with $\mathrm{NaCl}$ solution. Figure S2b shows the EDS analysis of the alumina nanotube channel after DI water rinse. This indicates that no $\mathrm{NaCl}$ ions remained in the alumina nanotube channel after the DI rinse. 